\title{
Explicit units and the equivariant Tamagawa number conjecture, II
}

\author{
David Burns* and Anthony Hayward
}

\begin{abstract}
We show that a special case of the equivariant Tamagawa number conjecture implies explicit restrictions on the structure of ideal class groups that are in general much finer than those which can be obtained by using the method of Thaine.
\end{abstract}

Mathematics Subject Classification (2000). 11R33.

Keywords. Equivariant Tamagawa number conjecture, ideal class groups.

\section{Introduction}

In [1], Bley and the first-named author showed that a particular case of the equivariant Tamagawa number conjecture of [9, Conjecture 4] implies, in the setting of Galois extensions of number fields, the existence of elements satisfying a variety of rather explicit 'Stark-type' conditions. More recently, the relevant special case of [9, Conjecture 4] has been verified for absolutely abelian fields [11] and an analogous conjecture for global function fields has been verified at all primes different from the residue characteristic [6]. In addition, the approach of [1] has been developed in [5], [6], [7] to prove refinements and generalisations of certain much-studied 'refined abelian Stark conjectures' that are due to inter alia Gross, Rubin and Tate.

The main insight of the present article (in Theorem 3.1) is that the approach of [1] can in certain cases be further refined to show that the Tamagawa number formalism predicts explicit structural information about ideal class groups that is in general very much finer than that which can be obtained by applying the celebrated method of Thaine [26] (as already used to spectacular effect by Rubin in [23]). Indeed, the original motivation behind this article was some striking recent work of Greither and Kučera in [16] proving annihilation statements for the class groups of a restricted family of absolutely abelian fields, improving upon those that can be obtained by the method of Thaine in that the 'special elements' involved are much better adapted to

\footnotetext{
${ }^{*}$ Supported by a Leverhulme Research Fellowship.
} 
the purpose at hand than are the Sinnott circular units which Thaine's method would use. In fact, the special elements in [16] arise by 'dividing' the Sinnott circular units by certain augmentation elements of the associated integral Galois group ring, and are constructed by means of rather involved combinatorial methods.

By contrast, in this article we shall combine some well known techniques and results of homological algebra with the general approach of [1] to show that the existence of the special elements constructed by Greither and Kučera in [16] is directly implied by the validity of the relevant case of [9, Conjecture 4]. By replacing the direct use of elaborate combinatorial methods with a reduction to the general formalism of [1], we obtain several rather concrete advantages. For example, our approach applies equally well in the setting of global function fields, as well as to extensions for which the associated $L$-functions have multiple-order zeroes at $s=0$ (rather than only first-order zeroes, as in [16]), in which case it predicts the existence of suitable 'systems' of special elements, and it also allows us to answer a question explicitly raised by Greither and Kučera in [16] by showing that the special element described in [loc. cit., Theorem 1] can be directly related to the Fitting ideals, rather than the annihilators (as in [loc. cit., Theorem 2]), of certain natural class groups (for more details see Remark 3.3). At the end of the article we also construct an explicit family of extensions showing that the cyclicity hypothesis used by Greither and Kučera in loc. cit. is indeed necessary in order to guarantee the existence of suitable special elements.

It is a pleasure for us to thank Manuel Breuning, Cornelius Greither and Radan Kučera for a number of helpful conversations and observations. We are also grateful to the referee for several suggestions which helped to improve the exposition.

In the sequel all unadorned tensor products are to be regarded as computed in the category of (complexes of) abelian groups.

\section{The leading term conjecture}

2.1. Formulation of the conjecture. Let $K / k$ be a nontrivial abelian extension of global fields with Galois group $G$. Let $S$ be a finite non-empty set of places of $k$ containing all archimedean places (if any) and all those that ramify in the extension $K / k$. We write $Y_{K, S}$ for the free abelian group on the set of places $S(K)$ of $K$ which lie above those in $S$ and $X_{K, S}$ for the kernel of the homomorphism $Y_{K, S} \rightarrow \mathbb{Z}$ that sends each element of $S(K)$ to 1 . We write $\mathcal{O}_{K, S}$ for the ring of $S(K)$-integers in $K$ and we set $U_{K, S}:=\operatorname{Spec}\left(\mathcal{O}_{K, S}\right)$ and $A_{K, S}:=\operatorname{Pic}\left(\mathcal{O}_{K, S}\right)$.

We define the $S$-truncated $\mathbb{C}[G]$-valued $L$-function of $K / k$ by setting

$$
\theta_{K / k, S}(s):=\sum_{\chi} L_{K / k, S}\left(s, \chi^{-1}\right) e_{\chi}
$$


where the index $\chi$ runs through all elements of $\operatorname{Hom}\left(G, \mathbb{C}^{\times}\right), L_{K / k, S}(s, \chi)$ denotes the $S$-truncated Artin $L$-function and $e_{\chi}:=(1 / \# G) \sum_{g \in G} \chi(g) g^{-1}$ is the idempotent at $\chi$. Its leading term at $s=0$ is then $\theta_{K / k, S}^{*}(0):=\sum_{\chi} L_{K / k, S}^{*}\left(0, \chi^{-1}\right) e_{\chi}$, where $L_{K / k, S}^{*}(0, \chi)$ is the leading coefficient in the Taylor expansion of $L_{K / k, S}(s, \chi)$ at 0. It is easily shown that $\theta_{K / k, S}^{*}(0)$ belongs to $\mathbb{R}[G]$, and in the remainder of this section we recall a conjectural description of the $\mathbb{Z}[G]$-submodule of $\mathbb{R}[G]$ that is generated by $\theta_{K / k, S}^{*}(0)$.

The key ingredient in this conjectural description is the determinant of a perfect complex of $\mathbb{Z}[G]$-modules that is described in the following result. In order to state this result we write $\mathfrak{D}(\mathbb{Z}[G])$ for the derived category of the abelian category of $G$ modules. For any object $C^{\bullet}$ of $\mathfrak{D}(\mathbb{Z}[G])$ with differential $d^{i}$ in each degree $i$ and any integer $m$ we write $C^{\bullet}[m]$ for the complex which is equal to $C^{i+m}$ in each degree $i$ and for which the differential in degree $i$ is equal to $(-1)^{m} d^{i+m}$. For any $G$-module $M$ and integer $m$ we write $M[m]$ for the complex which is equal to $M$ in degree $-m$ and is equal to 0 in all other degrees.

In the following result we use the complex $R \Gamma_{c \text {,ét }}\left(U_{K, S}, \mathbb{Z}\right)$ that is defined in $[8,(3)]$. We recall that this complex is an object of $\mathfrak{D}(\mathbb{Z}[G])$ which computes the cohomology 'with compact support' of the constant étale sheaf $\mathbb{Z}$ on $U_{K, S}$.

Lemma 2.1. There exists a complex $\tilde{\Psi}_{S}^{\bullet}$ of $\mathbb{Z}[G]$-modules of the form

$$
\Psi_{S}^{0} \stackrel{d}{\longrightarrow} \Psi_{S}^{1} \longrightarrow X_{K, S} \otimes \mathbb{Q}
$$

which has both of the following properties.

(i) There exists a distinguished triangle in $\mathfrak{D}(\mathbb{Z}[G])$ of the form

$$
\tilde{\Psi}_{S}^{\bullet} \rightarrow \operatorname{Hom}_{\mathbb{Z}}\left(R \Gamma_{c, \text { ét }}\left(U_{K, S}, \mathbb{Z}\right), \mathbb{Q} / \mathbb{Z}[-3]\right) \rightarrow \hat{\mathcal{O}}_{K, S}^{\times} / \mathcal{O}_{K, S}^{\times}[0] \rightarrow \tilde{\Psi}_{S}^{\bullet}[1]
$$

where $\hat{\mathcal{O}}_{K, S}^{\times}$denotes the profinite completion of $\mathcal{O}_{K, S}^{\times}$and the second arrow is the unique morphism in $\mathfrak{D}(\mathbb{Z}[G]$ ) which induces upon cohomology (in degree 0 ) the composite of the canonical identification of

$$
H^{0}\left(\operatorname{Hom}_{\mathbb{Z}}\left(R \Gamma_{c, \text { ét }}\left(U_{K, S}, \mathbb{Z}\right), \mathbb{Q} / \mathbb{Z}[-3]\right)\right)
$$

with $\hat{\mathcal{O}}_{K, S}^{\times}$and the natural projection from $\hat{\mathcal{O}}_{K, S}^{\times}$to $\hat{\mathcal{O}}_{K, S}^{\times} / \mathcal{O}_{K, S}^{\times}$.

(ii) $\Psi_{S}^{0}$ is a finitely generated cohomologically-trivial G-module, $\Psi_{S}^{1}$ is a finitely generated free $\mathbb{Z}[G]$-module, and the distinguished triangle in claim (i) induces exact sequences of the form

$$
\begin{gathered}
0 \longrightarrow \mathcal{O}_{K, S}^{\times} \longrightarrow \Psi_{S}^{0} \stackrel{d}{\longrightarrow} \Psi_{S}^{1} \longrightarrow X_{K, S}^{\prime} \longrightarrow 0 \\
0 \longrightarrow A_{K, S} \longrightarrow X_{K, S}^{\prime} \longrightarrow X_{K, S} \longrightarrow 0 .
\end{gathered}
$$


Proof. An explicit description of the complex $\operatorname{Hom}_{\mathbb{Z}}\left(R \Gamma_{c \text {,ét }}\left(U_{K, S}, \mathbb{Z}\right), \mathbb{Q} / \mathbb{Z}[-3]\right)$ allows to easily deduce the existence of a complex $\tilde{\Psi}_{S}^{\bullet}$ with all of the properties stated above. Indeed, if $A_{K, S}$ is trivial, then this is the method used by Flach and the firstnamed author in [8, Proposition 3.1] to prove the existence of just such a complex $\tilde{\Psi}_{S}^{\bullet}$ (we note that whilst the argument of loc. cit. is phrased solely in terms of number fields, it extends immediately to the case of global function fields). Further, in the general case the explicit computation of $\operatorname{Hom}_{\mathbb{Z}}\left(R \Gamma_{c \text {,ét }}\left(U_{K, S}, \mathbb{Z}\right), \mathbb{Q} / \mathbb{Z}[-3]\right)$ that is given in [loc. cit., pp. 1356-1358] can be completed in exactly the same way even if $A_{K, S}\left(\cong H_{\mathrm{et}}^{1}\left(U_{K, S}, \mathbb{G}_{m}\right)\right)$ is non-trivial and this leads directly to the existence of a complex $\tilde{\Psi}_{S}^{\bullet}$ with all of the required properties.

For any commutative ring $R$ we write $\operatorname{Det}_{R}$ for the determinant functor of Knudsen and Mumford [19], valued in the category $\mathcal{P}(R)$ of graded invertible $R$-modules, and for any object $(\mathcal{L}, r)$ of $\mathcal{P}(R)$ we set $(\mathscr{L}, r)^{-1}:=\left(\operatorname{Hom}_{R}(\mathcal{L}, R),-r\right)$ (which is again an object of $\mathcal{P}(R))$.

In terms of the notation of Lemma 2.1 we write $\Psi_{S}^{\bullet}$ for the perfect complex of $\mathbb{Z}[G]$-modules which is equal to $\Psi_{S}^{0} \stackrel{d}{\rightarrow} \Psi_{S}^{1}$, where the first term is placed in degree 0 and the cohomology groups are identified with $\mathcal{O}_{K, S}^{\times}$and $X_{K, S}^{\prime}$ by means of (1). Then we obtain an isomorphism

$$
\vartheta_{\Psi_{S}^{\bullet}, \mathbb{R}}: \operatorname{Det}_{\mathbb{R}[G]}\left(\Psi_{S}^{\bullet} \otimes \mathbb{R}\right) \cong(\mathbb{R}[G], 0)
$$

in $\mathcal{P}(\mathbb{R}[G])$ by composing the isomorphism

$$
\operatorname{Det}_{\mathbb{R}[G]}\left(\Psi_{S}^{\bullet} \otimes \mathbb{R}\right) \cong \operatorname{Det}_{\mathbb{R}[G]}\left(\mathcal{O}_{K, S}^{\times} \otimes \mathbb{R}\right) \otimes_{\mathcal{P}(\mathbb{R}[G])} \operatorname{Det}_{\mathbb{R}[G]}\left(X_{K, S} \otimes \mathbb{R}\right)^{-1}
$$

that is induced by the scalar extension of (1) with the map $\operatorname{Det}_{\mathbb{R}[G]}\left(\operatorname{Reg}_{K, S}\right) \otimes_{\mathcal{P}(\mathbb{R}[G])}$ id and then the evaluation pairing on the space $\operatorname{Det}_{\mathbb{R}[G]}\left(X_{K, S} \otimes \mathbb{R}\right)$, where $\operatorname{Reg}_{K, S}$ denotes the $\mathbb{R}[G]$-equivariant isomorphism $\mathcal{O}_{K, S}^{\times} \otimes \mathbb{R} \rightarrow X_{K, S} \otimes \mathbb{R}$ which at each element $u$ of $\mathcal{O}_{K, S}^{\times}$satisfies

$$
\operatorname{Reg}_{K, S}(u)=-\sum_{w \in S(K)} \log |u|_{w} \cdot w,
$$

with $|\cdot|_{w}$ denoting the normalised absolute value of each place $w$.

Conjecture $\mathbf{C}(\boldsymbol{K} / \boldsymbol{k})$. One has an equality in $\mathcal{P}(\mathbb{Z}[G])$ of the form

$$
\left(\theta_{K / k, S}^{*}(0) \cdot \mathbb{Z}[G], 0\right)=\vartheta_{\Psi_{S}^{\bullet}, \mathbb{R}}\left(\operatorname{Det}_{\mathbb{Z}[G]} \Psi_{S}^{\bullet}\right) .
$$

Remark 2.2. Under the conditions of Lemma 2.1, the complex $\Psi_{S}^{\bullet}$ is unique to within an isomorphism in $\mathfrak{D}(\mathbb{Z}[G])$ that induces the identity map on all (non-zero) degrees of cohomology, and this can be used to show that the (graded) lattice $\vartheta_{\Psi_{S}, \mathbb{R}}\left(\operatorname{Det}_{\mathbb{Z}[G]} \Psi_{S}^{\bullet}\right)$ depends only upon the pair $(K / k, S)$. 
Remark 2.3. The same argument as used to prove [4, Theorem 2.1.2 (i)] shows that the validity of Conjecture $\mathrm{C}(K / k)$ is independent of the chosen set $S$. Further, if $S$ is large enough to ensure that $A_{K, S}$ vanishes, then $\Psi_{S}^{\bullet}$ coincides with the complex described in [loc. cit., Proposition 2.1.1] and [8, Remark following Proposition 3.1] (in the number field case) and also in [5, §2]. It follows that Conjecture $\mathrm{C}(K / k)$ coincides with the conjectures studied in [4], [8], and is therefore equivalent (in the number field case) to the 'Equivariant Tamagawa Number Conjecture' of [9, Conjecture 4(iv)] as applied to the pair $\left(h^{0}(\operatorname{Spec} K), \mathbb{Z}[G]\right)$, where $h^{0}(\operatorname{Spec} K)$ is considered as a motive that is defined over $k$ and has coefficients $\mathbb{Q}[G]$ (see [4, Theorem 2.4.1] or [10, §3] for different proofs of this fact). Since $G$ is abelian, this observation shows that Conjecture $\mathrm{C}(K / k)$ implies the relevant special case of the 'Generalised Iwasawa Main Conjecture' formulated by Kato in [18, §3.2] (cf. [10, $\S 2]$ in this regard). In addition, one knows that Conjecture $\mathrm{C}(K / k)$ is valid if either $k=\mathbb{Q}$ (cf. [11, Theorem 8.1, Remark 8.1], [14, Theorem 5.1]) or $k$ is a global function field whose characteristic is coprime to the order of $G$ [6, Corollary 1, Remark 5].

Remark 2.4. If $K / k$ is an extension of global function fields, then $\Psi_{S}^{\bullet}$ can also be interpreted in terms of the Weil-étale cohomology of $\mathbb{G}_{m}$ on $U_{K, S}[6, \S 2.2]$. In particular, if $K=k$ is a global function field, then one can show that Conjecture $\mathrm{C}(K / k)$ is equivalent to a special case of the conjecture formulated by Lichtenbaum in [20, Conjecture 8.1e)].

2.2. An explicit reinterpretation. In this section we impose certain additional hypotheses on $K / k$ in order to give an interpretation of Conjecture $\mathrm{C}(K / k)$ in terms of the existence of $S$-units satisfying a variety of explicit conditions. This reinterpretation arises by combining the approach of Bley and the first-named author in [1] with that of Rubin in [24] and will be used in the next section to prove our main result.

For convenience we now label, and hence order, the elements of $S$ as $\left\{v_{i}: 0 \leq\right.$ $i \leq n\}$. For each such index $i$ we choose a place $w_{i}$ of $K$ above $v_{i}$, we let $G_{i}$ denote the decomposition group of $w_{i}$ in $G$, and we set $K_{i}:=K^{G_{i}}$. For any place $w$ of $K$ and a subfield $L$, we write $w_{L}$ for the place of $L$ induced by $w$, and we abbreviate $\left(w_{i}\right)_{L}$ by $w_{i, L}$. We assume throughout that there exists a strictly positive integer $r$ with $r<n$ which is such that $G_{i}$ is trivial for each index $i$ with $1 \leq i \leq r$.

We write $r(\chi)$ for the order of vanishing of the meromorphic function $L_{S}(s, \chi)$ at $s=0$.

Lemma 2.5. For each nontrivial character $\chi$ of $G$, the integer $r(\chi)$ is equal to the number of places $v_{i}$ in $S$ for which $\chi$ is trivial on $G_{i}$.

If $\chi$ is the trivial character, then $r(\chi)$ is equal to $n$.

Proof. This follows immediately from [25, Chapter I, Proposition I.3.4]. 
For each prime $p$ we write $\mathbb{Z}_{(p)}$ for the $p$-localisation of $\mathbb{Z}$.

Lemma 2.6. Assume that $\# G$ is coprime to both $\#\left(K^{\times}\right)_{\text {tors }}$ and $\# A_{K, S}$ and that Conjecture $\mathrm{C}(K / k)$ is valid. Assume also that $G_{0}=G$, that $G_{i}$ is trivial if $1 \leq i \leq r$ and that $G_{i}$ is cyclic if $r<i \leq n$. For each integer $i$ with $r<i \leq n$ fix a generating element $g_{i}$ of $G_{i}$.

Then for each $j$ with $1 \leq j \leq n$ there exists an element $\epsilon_{j}$ of $\mathcal{O}_{K_{j}, S}^{\times}$which satisfies all of the following conditions.

(i) If $\mathcal{E}_{S}$ denotes the $G$-submodule of $\mathcal{O}_{K, S}^{\times}$that is generated by $\left\{\epsilon_{j}: 1 \leq j \leq n\right\}$, then the index $\left(\mathcal{O}_{K, S}^{\times}: \mathcal{E}_{S}\right)$ is finite and coprime to $\# G$.

(ii) If $r<j \leq n$, then for each place $w$ of $K$ the local symbol $\left(\epsilon_{j}, K_{w} /\left(K_{j}\right)_{w_{K_{j}}}\right)$ is equal to $g_{j}$ if $w$ is equal to $w_{j}$ and is equal to the identity element in all other cases.

(iii) Let $M$ denote the $n \times n$ matrix in $\mathbb{R}[G]$ which has $(i, j)$-th entry equal to

$$
M_{i j}:= \begin{cases}y_{i j}, & \text { if } 1 \leq i, j \leq r, \\ y_{i j}+\delta_{i j}\left(g_{i}-1\right), & \text { otherwise }\end{cases}
$$

where $y_{i j}$ denotes the unique element of $\mathbb{R}[G] \cdot \sum_{g \in G_{j}} g$ which satisfies

$$
\frac{1}{\# G_{i}} \operatorname{Reg}_{K, S}\left(\epsilon_{i}\right)=\sum_{j=1}^{j=n} y_{i j}\left(w_{j}-w_{0}\right)
$$

and $\delta_{i j}$ is equal to 1 if $i=j$ and is equal to 0 otherwise. Then $\operatorname{Fitt}_{\mathbb{Z}[G]}\left(A_{K, S}\right)$ is an invertible ideal of $\mathbb{Z}[G]$ and there exists an element $x$ of $\bigcap_{p \mid \# G} \mathbb{Z}_{(p)}[G]^{\times}$ which satisfies both

$$
\theta_{K / k, S}^{*}(0)=x \operatorname{det}(M) \text { and } \mathbb{Z}[G] x^{-1}=\operatorname{Fitt}_{\mathbb{Z}[G]}\left(\mathcal{O}_{K, S}^{\times} / \mathcal{E}_{S}\right) \operatorname{Fitt}_{\mathbb{Z}[G]}\left(A_{K, S}\right)^{-1} .
$$

Proof. This is merely a slight elaboration of the main results of Bley and the firstnamed author in [1] and hence follows directly from an explicit computation of the image of Det $\operatorname{De}_{\mathbb{Z}[G]} \Psi_{S}^{\bullet}$ under the map $\vartheta_{\Psi_{S}^{\bullet}, \mathbb{R}}$. Indeed, under the stated hypotheses on $K / k$ and $S$, the existence of a set of elements $\left\{\epsilon_{j}: 1 \leq j \leq n\right\}$ satisfying claims (i) and (ii) is proved in [loc. cit., Theorem 3.2a), b)] and then, given the definition of the matrix $M$ in claim (iii), the existence of an element $x$ of $\mathbb{Q}[G]^{\times}$which satisfies the last two displayed equalities of claim (iii) follows from the argument of [loc. cit., Theorem 2.3] (any interested reader can find more details as to the latter deduction in $[6$, Proposition $6.1,(19),(20)])$. It therefore only remains for us to show that any such element $x$ must belong to $\mathbb{Z}_{(p)}[G]^{\times}$for each $p$ dividing \#G. But this follows 
because for each such $p$ claim (i) combines with our assumption that $\# G$ is coprime to \# $A_{K, S}$ to imply that

$$
\begin{aligned}
Z_{(p)}[G] x^{-1} & =\mathbb{Z}[G] x^{-1} \otimes \mathbb{Z}_{(p)} \\
& =\operatorname{Fitt}_{\mathbb{Z}_{(p)}[G]}\left(\left(\mathcal{O}_{K, S}^{\times} / \mathcal{E}_{S}\right) \otimes \mathbb{Z}_{(p)}\right) \operatorname{Fitt}_{\mathbb{Z}_{(p)}[G]}\left(A_{K, S} \otimes \mathbb{Z}_{(p)}\right)^{-1} \\
& =\operatorname{Fitt}_{\mathbb{Z}_{(p)}[G]}(0) \operatorname{Fitt}_{\mathbb{Z}_{(p)}[G]}(0)^{-1}=\mathbb{Z}_{(p)}[G] .
\end{aligned}
$$

We now consider an intermediate field $L=K^{H}$ between $K$ and $k$ and set $\Gamma:=$ $\operatorname{Gal}(L / k) \cong G / H$. We assume that the ordering of $S$ is such that there exists an integer $r_{L}$ such that $v_{i}$ is totally split in $L$ (or equivalently $G_{i} \subseteq H$ ) if $1 \leq i \leq r_{L}$ and that $v_{i}$ is not totally split in $L$ if $r_{L}<i \leq n$. Note that this implies $r_{L} \geq r$. Following the approach of [24], we observe that there exists a unique element $\eta_{L, S}$ of the space $\mathbb{R} \otimes \bigwedge_{\mathbb{Z}[\Gamma]}^{r_{L}} \mathcal{O}_{L, S}^{\times}$which satisfies

$$
\left(\bigwedge_{\mathbb{R}[\Gamma]}^{r_{L}} \operatorname{Reg}_{L, S}\right)\left(\eta_{L, S}\right)=\lim _{s \rightarrow 0} s^{-r_{L}} \theta_{L / k, S}(s) \cdot \bigwedge_{i=1}^{i=r_{L}}\left(w_{i, L}-w_{0, L}\right)
$$

and is such that for each element $\chi$ of $\operatorname{Hom}\left(\Gamma, \mathbb{C}^{\times}\right)$one has $e_{\chi}\left(\eta_{L, S}\right) \neq 0$ if and only if $e_{\chi} \cdot \lim _{s \rightarrow 0} s^{-r_{L}} \theta_{L / k, S}(s) \neq 0$.

Proposition 2.7. Assume the hypotheses of Lemma 2.6 and the previous paragraph. Then in $\mathbb{Q} \otimes \bigwedge_{\mathbb{Z}[\Gamma]}^{r_{L}} \mathcal{O}_{L, S}^{\times}$we have

$$
\eta_{L, S}=x \prod_{j=r_{L}+1}^{j=n}\left(g_{j}-1\right) \cdot \bigwedge_{i=1}^{i=r_{L}} N_{K_{i} / K_{i} \cap L} \epsilon_{i} .
$$

Proof. Let $e_{H}=\frac{1}{\# H} \sum_{h \in H} h$, and write $q_{H}: \mathbb{R}[G] e_{H} \rightarrow \mathbb{R}[\Gamma]$ for the $\mathbb{R}[G]-$ isomorphism which sends $e_{H}$ to 1 . The complex characters of $\Gamma$ are in bijection with those characters of $G$ which are trivial on $H$, and [25, 4.2.3] shows that $L_{K / k, S}\left(s, \operatorname{Inf}_{\Gamma}^{G}(\chi)\right)=L_{L / k, S}(s, \chi)$ for each such character $\chi$. The criteria of Lemma 2.5 show that $L_{L / k, S}(s, \chi)$ vanishes at $s=0$ to order at least $r_{L}$, and to order exactly $r_{L}$ if $\operatorname{Inf}_{\Gamma}^{G} \chi$ is nontrivial on each of the subgroups $G_{r_{L}+1}, \ldots, G_{n}$. We write $e:=\sum_{\chi} e_{\operatorname{Inf}_{\Gamma}^{G}(\chi)}$, where the sum is over these $\chi$ where the order of vanishing is exactly $r_{L}$. Then, setting $\theta_{L / k, S}^{r_{L}}(0):=\lim _{s \rightarrow 0} s^{-r_{L}} \theta_{L / k, S}(s)$, Lemma 2.6 (iii) shows that

$$
\theta_{L / k, S}^{r_{L}}(0)=q_{H}(x e \operatorname{det}(M)) .
$$

For each integer $i$ with $0 \leq i \leq n$ we set $T_{i}:=\sum_{g \in G_{i}} g \in \mathbb{Z}[G]$. Then we may write $M$ in the block form

$$
M=Y+\left(\begin{array}{l|l}
0 & 0 \\
\hline 0 & \Delta
\end{array}\right)
$$


where $Y$ has $(i, j)$-th entry $y_{i j} \in \mathbb{R}[G] \cdot T_{j}$ and $\Delta$ is the $\left(n-r_{L}\right) \times\left(n-r_{L}\right)$ diagonal matrix with $(j, j)$-th entry $g_{r_{L}+j}-1$ for $1 \leq j \leq n-r_{L}$.

Lemma 2.5 shows that if $j$ satisfies $r_{L}<j \leq n$ then $e T_{j}=0$, and so $e y_{i j}=0$ for all $i$ and $j$ with $1 \leq i \leq n$ and $r_{L}<j \leq n$. Therefore the matrix $e M$ takes the form

$$
e M=\left(\begin{array}{c|c}
M^{\prime} & * \\
\hline 0 & e \Delta
\end{array}\right)
$$

where $M^{\prime}$ is the $r_{L} \times r_{L}$ matrix with $(i, j)$-th entry $e y_{i j}$. Set $t_{S}:=\prod_{j=r_{L}+1}^{j=n}\left(g_{j}-1\right) \in$ $\mathbb{Z}[\Gamma]$; here the product is understood to be the identity element of $\mathbb{Z}[\Gamma]$ if $r_{L}=n$. We then have $e \operatorname{det}(M)=t_{S} \operatorname{det}\left(M^{\prime}\right)$. Now

$$
\operatorname{det}\left(M^{\prime}\right) \cdot \bigwedge_{i=1}^{i=r_{L}}\left(w_{i, L}-w_{0, L}\right)=\bigwedge_{i=1}^{i=r_{L}}\left(\sum_{j=1}^{j=r_{L}} e y_{i j}\left(w_{j, L}-w_{0, L}\right)\right),
$$

and noting that $e\left(w_{j, L}-w_{0, L}\right)=0$ if $j>r_{L}$, the right hand side of this equation is equal to $e \bigwedge_{i=1}^{i=r_{L}}\left(\sum_{j=1}^{n} y_{i j}\left(w_{j, L}-w_{0, L}\right)\right)$.

The natural inclusion map $\mathcal{O}_{L, S}^{\times} \rightarrow \mathcal{O}_{K, S}^{\times}$fits into a commutative diagram

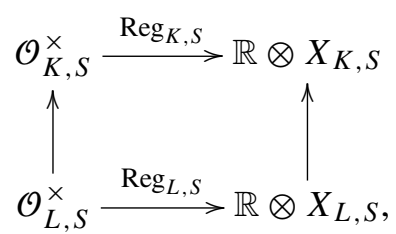

in which the map $\mathbb{R} \otimes X_{L, S} \rightarrow \mathbb{R} \otimes X_{K, S}$ sends $w_{L}$ to $\left(\# H e_{H}\right) w$; see [25, bottom of p. 29]. Combining this with (3) implies

$$
\operatorname{Reg}_{L, S}\left(N_{K_{i} / K_{i} \cap L} \epsilon_{i}\right)=\sum_{j=1}^{j=n} y_{i j}\left(w_{j, L}-w_{0, L}\right)
$$

for each index $i$ with $1 \leq i \leq r_{L}$, and so we conclude that

$$
\begin{aligned}
\operatorname{det}\left(M^{\prime}\right) \cdot \bigwedge_{i=1}^{i=r_{L}}\left(w_{i, L}-w_{0, L}\right) & =e \cdot \bigwedge_{i=1}^{i=r_{L}} \operatorname{Reg}_{L, S}\left(N_{K_{i} / K_{i} \cap L} \epsilon_{i}\right) \\
& =\left(\bigwedge_{\mathbb{R}[\Gamma]}^{r_{L}} \operatorname{Reg}_{L, S}\right)\left(e \cdot \bigwedge_{i=1}^{i=r_{L}} N_{K_{i} / K_{i} \cap L} \epsilon_{i}\right) .
\end{aligned}
$$

Hence we obtain

$$
\begin{aligned}
\theta_{L / k, S}^{r_{L}}(0) \cdot \bigwedge_{i=1}^{i=r_{L}}\left(w_{i, L}-w_{0, L}\right) & =x e \operatorname{det}(M) \cdot \bigwedge_{i=1}^{i=r_{L}}\left(w_{i, L}-w_{0, L}\right) \\
& =\operatorname{xet}_{S} \cdot\left(\bigwedge_{\mathbb{R}[\Gamma]}^{r_{L}} \operatorname{Reg}_{L, S}\right)\left(\bigwedge_{i=1}^{i=r_{L}} N_{K_{i} / K_{i} \cap L} \epsilon_{i}\right) .
\end{aligned}
$$

To establish the equality of Proposition 2.7 it is thus enough to show that $e t_{S}=t_{S}$. If $r_{L}=n$, we have $e=1$ and so $e t_{S}=t_{S}$ is clear. If $r_{L}<n$ then, for each character $\chi$ inflated from $\Gamma$ to $G$ with $r(\chi)>r_{L}$, we have $\chi\left(t_{S}\right)=\prod_{j=r_{L}+1}^{j=n}\left(\chi\left(g_{j}\right)-1\right)=0$, since a factor in the product must be 0 by Lemma 2.5. This shows that $e t_{S}=t_{S}$ in both cases and hence completes the proof. 


\section{Special cases}

In this section we assume the notation and hypotheses of Lemma 2.6. We also fix a prime $p$ and an integer $\ell>1$ which is a power of $p$.

We assume given a cyclic extension $K / k$ of degree $\ell$, with $\# k_{\text {tors }}^{\times}$not divisible by $p$. We set $G:=\operatorname{Gal}(K / k)$ and fix a generating element $\sigma$ of $G$. We let $\mathrm{T}=\sum_{g \in G} g$ be the norm element in the integral group ring $\mathbb{Z}[G]$ and we denote the corresponding quotient ring $\mathbb{Z}[G] / \mathbb{Z}[G] \cdot \mathrm{T}$ by $\mathcal{A}$. We also set $e:=1-\frac{1}{\ell} \mathrm{T}$ and observe that the $G$-equivariant homomorphism $\mathbb{Z}[G] e \rightarrow \mathcal{A}$ which takes $e$ to 1 is a ring isomorphism. We set $\mathcal{A}_{(p)}:=\mathcal{A} \otimes \mathbb{Z}_{(p)}$.

For any $G$-module $M$ we let $M^{\mathrm{T}=0}$ denote the kernel of the endomorphism of $M$ that is induced by the action of T. We also write $\bar{M}$ for the quotient of $M$ by its $\mathbb{Z}$-torsion submodule $M_{\text {tors }}$ and we regard $\bar{M}$ as a submodule of the space $M \otimes \mathbb{Q}$ in the natural way.

For each integer $i$ with $0 \leq i \leq n$ we define a homomorphism $f_{i}: \mathcal{O}_{k, S}^{\times} \rightarrow \mathbb{Z} / \ell \mathbb{Z}$ by the condition that the local reciprocity symbol $\left(u, K_{w_{i}} / k_{v_{i}}\right)$ is equal to $\sigma^{f_{i}(u)}$ for all elements $u$ of $\mathcal{O}_{k, S}^{\times}$. We observe that the group $\mathbb{Z} / \ell \mathbb{Z}$ acts on any group 'modulo $\ell$ ', meaning modulo $\ell$-th powers.

Theorem 3.1. Assume that $p \nmid \# k_{\text {tors }}^{\times}$and let $K / k$ be a cyclic extension of order $\ell$ and group $G$. Let $S=\left\{v_{0}, \ldots, v_{n}\right\}$ be a set of places of $k$ containing all archimedean places and all which ramify in $K / k$ and for each index $i$ write $G_{i}$ for the decomposition subgroup of $v_{i}$ in $G$. We assume that $G_{0}=G$ and also that there exists an integer $r$ with $1 \leq r<n$ which is such that $G_{i}$ is trivial, resp. $G_{i}$ is equal to $G$, if $1 \leq i \leq r$, resp. $r<i \leq n$.

Suppose in addition that Conjecture $C(K / k)$ is valid. Then $\eta_{K, S}$ belongs to $\overline{\bigwedge_{\mathbb{Z}[G]}^{r} \mathcal{O}_{K, S}^{\times}} \otimes \mathbb{Z}_{(p)}$ by Proposition 2.7. Furthermore, there exists an element $\varepsilon$ of $\overline{\bigwedge_{\mathbb{Z}[G]}^{r} \mathcal{O}_{K, S}^{\times}} \otimes \mathbb{Z}_{(p)}$, respectively of $\overline{\bigwedge_{\mathbb{Z}[G]}^{r} \mathcal{O}_{K, S}^{\times}}$if $\eta_{K, S}$ belongs to $\overline{\bigwedge_{\mathbb{Z}[G]}^{r} \mathcal{O}_{K, S}^{\times}}$, which has all of the following properties.

(i) In $\mathbb{Q} \otimes \bigwedge_{\mathbb{Z}[G]}^{r} \mathcal{O}_{K, S}^{\times}$one has $\eta_{K, S}=(\sigma-1)^{n-r}(\varepsilon)$.

(ii) The congruence

$$
\left(\bigwedge_{\mathbb{Z}[G]}^{r} N_{K / k}\right)(\varepsilon) \equiv\left(f_{r+1} \wedge \cdots \wedge f_{n}\right)\left(\eta_{k, S}\right)
$$

holds in $\overline{\bigwedge_{\mathbb{Z}}^{r} \mathcal{O}_{k, S}^{\times}} \otimes \mathbb{Z}_{(p)}$ modulo $\ell$.

(iii) Let $S_{1}$ denote the subset $\left\{v_{i}: 1 \leq i \leq r\right\}$ of $S$ consisting of those places which have trivial decomposition group. Then $S_{1}$ contains all archimedean places of $k$. Further, there exists a free $\mathbb{Z}[G]$-submodule $\&$ of $\mathcal{O}_{K, S}^{\times}$of rank $r$ and an integer 
$t_{\mathscr{E}}$ which is a power of $p$ such that both $\bigwedge_{\mathbb{Z}[G]}^{r} \mathcal{E} \otimes_{\mathbb{Z}[G]} \mathcal{A}_{(p)}=t_{\mathcal{E}} \mathcal{A}_{(p)} \mathcal{E}$ and

$$
\begin{aligned}
t_{\mathcal{E}}^{-1}(\sigma-1)^{r} \cdot \operatorname{Fitt}_{\mathcal{A}}\left(\left(\mathcal{O}_{K, S_{1}}^{\times} / \mathcal{O}_{K, S_{1}}^{\times} \cap \mathcal{E}\right)^{\mathrm{T}=0}\right) \otimes \mathbb{Z}_{(p)} \subseteq \operatorname{Fitt}_{\mathbb{Z}[G]}\left(A_{K, S}\right) \mathcal{A}_{(p)} \\
\subseteq t_{\mathcal{E}}^{-1} \operatorname{Fitt}_{\mathcal{A}}\left(\left(\mathcal{O}_{K, S_{1}}^{\times} / \mathcal{O}_{K, S_{1}}^{\times} \cap \mathcal{E}\right)^{\mathrm{T}=0}\right) \otimes \mathbb{Z}_{(p)} .
\end{aligned}
$$

Further, if $r=1$, then we may take $t_{\mathcal{E}}=1$.

In the course of proving this result we shall actually obtain finer information than is provided by the inclusions given in claim (iii) (cf. Lemma 3.7). However, before giving the proof we use this result to answer a question raised by Greither and Kučera in [16]. To do this, we take $p$ odd, assume that $k=\mathbb{Q}$, and fix a set $\left\{p_{i}: 1 \leq i \leq n\right\}$ of distinct rational primes which are congruent to 1 modulo $\ell$, with $n \geq 2$. We then let $K / \mathbb{Q}$ be a cyclic extension of degree $\ell$, of Galois group $G=\langle\sigma\rangle$, in which each $p_{i}$ has full decomposition group and all other primes are unramified. Since such a field is totally real, the infinite place $\infty$ of $\mathbb{Q}$ splits completely in $K$, and so we set $r=1$ and $S=\left\{p_{1}, \infty, \ldots, p_{n}\right\}$, where the ordering of $S$ is chosen to be compatible with the assumptions of Theorem 3.1 (so $S_{1}=\{\infty\}$ in the notation of Theorem 3.1 (iii)). We note that Conjecture $\mathrm{C}(K / k)$ is valid by Remark 2.3. For each index $i$ we fix a complex $p_{i}$-th root of 1 , and call it $\zeta_{p_{i}}$. We denote the Sinnott circular unit of conductor level by $\eta:=\mathrm{N}_{\mathbb{Q}\left(\zeta_{p_{1} \cdots p_{n}}\right) / K}\left(1-\zeta_{p_{1}} \cdots \zeta_{p_{n}}\right)$.

Define an $n \times n$ matrix $A$ over $\mathbb{Z} / \ell \mathbb{Z}$ as follows. The field $K$ is contained in $\mathbb{Q}\left(\zeta_{p_{1} \cdots p_{n}}\right)$. If $i \neq j$, we let $a_{i j}$ be the unique class in $\mathbb{Z} / \ell \mathbb{Z}$ such that $\sigma^{a_{i j}}$ is the restriction of the automorphism $\zeta_{j} \mapsto \zeta_{j}^{p_{i}}$. Then choose $a_{i i}$ for each $i$ in such a way that the matrix has zero row sums. It is easy to see that this corresponds to the definition given by Greither and Kučera before [16, Theorem 1]. We define $A_{i}$ to be the lift to $\mathbb{Z}$ of the $(i, i)$ minor of $A$ satisfying $0<A_{i} \leq \ell{ }^{1}$

We let $\mathcal{O}_{K}$ denote the ring of algebraic integers $\mathcal{O}_{K, S_{1}}$ in $K$ and set $A_{K}:=$ $\operatorname{Pic}\left(\mathcal{O}_{K}\right)$.

Corollary 3.2. Let $K / \mathbb{Q}$ be a cyclic extension of order a power of an odd prime $p$ and assume that the decomposition group of each prime $p_{i}$ which ramifies in $K / \mathbb{Q}$ is equal to $G$. Set $S=\left\{p_{1}, \infty, \ldots, p_{n}\right\}$ (where the ordering of $S$ is chosen to be compatible with that used in Theorem 3.1). Then there exists an element $\varepsilon$ of $\mathcal{O}_{K, S}^{\times}$ which satisfies all of the following conditions.

(i) $(\sigma-1)^{n-1}(\varepsilon)=\eta$.

(ii) $\mathrm{N}_{K / \mathbb{Q}}(\varepsilon)^{(-1)^{n-1}}=\prod_{i=1}^{i=n} p_{i}^{A_{i}}$.

\footnotetext{
${ }^{1}$ Note that we have preferred $A_{i}=l$ to $A_{i}=0$. This will ensure that $\varepsilon$ is not a unit. Since $\varepsilon$ may be adjusted by rational factors, as explained in the proof of Corollary 3.2 below, any length $\ell$ range for $A_{i}$ may be specified.
} 
(iii)

$$
\begin{aligned}
(\sigma-1) \cdot \operatorname{Ann}_{\mathcal{A}}\left(\mathcal{O}_{K}^{\times} / \varepsilon^{\sigma-1}\right) \otimes \mathbb{Z}_{(p)} & \subseteq \operatorname{Fitt}_{\mathcal{A}}\left((\sigma-1) A_{K}\right) \otimes \mathbb{Z}_{(p)} \\
& \subseteq \operatorname{Ann}_{\mathcal{A}}\left(\mathcal{O}_{K}^{\times} / \varepsilon^{\sigma-1}\right) \otimes \mathbb{Z}_{(p)} .
\end{aligned}
$$

Remark 3.3. This answers the question raised in $[16$, Comment following Theorem 2], which asks about the possibility of using elements of the form $\varepsilon$ to produce bounds on the Fitting ideal Fitt $\mathcal{A}_{\mathcal{A}}\left((\sigma-1) A_{K}\right) \otimes \mathbb{Z}_{(p)}$.

In the remainder of this section we first prove the deduction of Corollary 3.2 from Theorem 3.1 and then prove Theorem 3.1 itself.

Proof of Corollary 3.2. To identify $\eta_{K, S}$ in (4) we must choose an infinite place of $K$ (" $w_{1, L}$ " in that formula); we use the place induced by the embedding of the overlying cyclotomic field into $\mathbb{C}$ by which $\zeta_{p_{1}} \cdots \zeta_{p_{n}}$ corresponds to $e^{2 \pi i /\left(p_{1} \cdots p_{n}\right)}$. The formulas for the values of $L$-functions of even Dirichlet characters show that $\eta_{K, S}^{2}=\eta$ in this situation, as in [17, §5]. Claim (i) of Theorem 3.2 shows that $\eta_{K, S}=(\sigma-1)^{n-1}\left(\varepsilon^{\prime}\right)$ for some element $\varepsilon^{\prime}$ of $\overline{\mathcal{O}_{K, S}^{\times}} \otimes \mathbb{Z}_{(p)}$. Since $\eta_{K, S}^{2}$ belongs to $\overline{\mathcal{O}_{K, S}^{\times}}$, setting $\varepsilon=\left(\varepsilon^{\prime}\right)^{2}$ gives $\pm \eta=(\sigma-1)^{n-1}(\varepsilon)$ in $\mathcal{O}_{K, S}^{\times}$. To see that the undetermined sign must be + , it is enough to take the norm to $\mathbb{Q}$ of both sides, and note that $N_{K / \mathbb{Q}} \eta=1$ by well-known properties of cyclotomic elements.

Now for the second assertion, we note that the analytic class number formula for $\mathbb{Q}$ implies that $\eta_{\mathbb{Q}, S}^{2}=p_{1} \wedge \cdots \wedge p_{n}$. Since $a_{i j}=-f_{j}\left(p_{i}\right)$, this shows that

$$
\left(f_{2} \wedge \cdots \wedge f_{n}\right)\left(\eta_{\mathbb{Q}, S}^{2}\right)=\prod_{i=1}^{i=n} p_{i}^{(-1)^{n-1} A_{i}}
$$

in $\mathbb{Z}_{S}^{\times}$modulo torsion and $\ell$-th powers. But the multiplicative torsion in $\mathbb{Z}_{S}$ consists of $\ell$-th powers since $\ell$ is odd. So Theorem 3.1 (ii) shows that

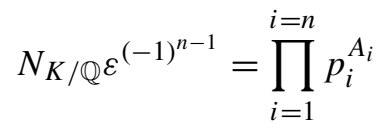

in $\mathbb{Z}_{S}^{\times}$modulo $\ell$-th powers. Now the transformation $\varepsilon \mapsto y \varepsilon$ for an arbitrary element $y$ of $\mathbb{Z}_{S}^{\times}$leaves claim (i) true and multiplies $N_{K / \mathbb{Q}} \varepsilon^{(-1)^{n-1}}$ by the $\ell$-th power of $y$. In this way $\varepsilon$ can be adjusted to achieve the equality of claim (ii) with the liftings $A_{i}$ in the specified range.

Note that the norm map $\mathrm{T}$ from $\mathcal{O}_{K}^{\times}$to $\mathbb{Z}^{\times}$is valued in a group of order 2 , and so is the zero map after tensoring with $\mathbb{Z}_{(p)}$. Therefore we may erase the ' $\mathrm{T}=0$ ' in Theorem 3.1 (iii). Further, the $\mathbb{Z}[G]$-module $\mathcal{E}$ there is the free submodule of $\mathcal{O}_{K, S}^{\times}$ 
generated by $\varepsilon$, and, since each finite prime in $S$ lies below a unique place of $K$, it is easy to see that $\mathcal{O}_{K}^{\times} \cap \mathcal{E}=\mathbb{Z}[G] \cdot \varepsilon^{\sigma-1}$. Hence

$$
\left(\mathcal{O}_{K}^{\times} / \mathcal{O}_{K}^{\times} \cap \mathcal{E}\right)^{\mathrm{T}=0}=\mathcal{O}_{K}^{\times} / \varepsilon^{\sigma-1}
$$

and this shows that Theorem 3.1 (iii) implies

$(\sigma-1) \cdot \operatorname{Ann}_{\mathcal{A}}\left(\mathcal{O}_{K}^{\times} / \varepsilon^{\sigma-1}\right) \otimes \mathbb{Z}_{(p)} \subseteq \operatorname{Fitt}_{\mathbb{Z}[G]}\left(A_{K, S}\right) \otimes \mathcal{A}_{(p)} \subseteq \operatorname{Ann}_{\mathcal{A}}\left(\mathcal{O}_{K}^{\times} / \varepsilon^{\sigma-1}\right) \otimes \mathbb{Z}_{(p)}$.

Our proof of Corollary 3.2 is therefore completed by means of the following observation.

Lemma 3.4. If $K / \mathbb{Q}$ and $S$ are as in Corollary 3.2, then the G-modules $A_{K, S}$ and $(\sigma-1) A_{K}$ are naturally isomorphic.

Proof. For any $G$-module $M$ we write $M^{G}$ for the submodule consisting of those elements which are fixed by the action of all elements of $G$. Then the exact sequence

$$
0 \longrightarrow A_{K}^{G} \stackrel{\subseteq}{\longrightarrow} A_{K} \stackrel{\times(\sigma-1)}{\longrightarrow}(\sigma-1) A_{K} \longrightarrow 0,
$$

reduces us to showing that $A_{K}^{G}$ is generated by the classes of primes above those in $S$.

Let $I_{K}$, resp. $P_{K}$, denote the group of fractional ideals, resp. principal fractional ideals, of $\mathcal{O}_{K}$. We claim that the natural morphism $I_{K}^{G} \rightarrow A_{K}^{G}$ is surjective. To show this we combine the cohomology sequence of the exact sequence $0 \rightarrow P_{K} \rightarrow$ $I_{K} \rightarrow A_{K} \rightarrow 0$ with the fact that $H^{1}\left(G, I_{K}\right)$ vanishes to obtain an exact sequence of the form $I_{K}^{G} \rightarrow A_{K}^{G} \rightarrow H^{1}\left(G, P_{K}\right) \rightarrow 0$. On the other hand, we may deduce that $H^{1}\left(G, P_{K}\right)$ vanishes by combining the cohomology sequence of $0 \rightarrow \mathcal{O}_{K}^{\times} \rightarrow$ $K^{\times} \rightarrow P_{K} \rightarrow 0$ together with the fact that $H^{1}\left(G, K^{\times}\right)$vanishes (by Hilbert 90) and $H^{2}\left(G, \mathcal{O}_{K}^{\times}\right) \cong \hat{H}^{0}\left(G, \mathcal{O}_{K}^{\times}\right)$vanishes (since $G$ is a cyclic group of odd order).

We now take an ideal $\mathfrak{a}:=\prod_{\mathfrak{p} \in S} \mathfrak{p}^{a_{\mathfrak{p}}} \prod \mathfrak{q} \in I_{K}^{G}$ where no prime ideal $\mathfrak{q}$ ramifies in $K / \mathbb{Q}$. Then $\prod \mathfrak{q}$ belongs to $I_{K}^{G}$ and so is of the form $m \mathcal{O}_{K}$ for some rational number $m$. It follows that $\mathfrak{a}$ and $\prod_{\mathfrak{p} \in S} \mathfrak{p}^{a_{\mathfrak{p}}}$ have the same image in $A_{K}$, as required.

Proof of Theorem 3.1. Let $r^{\prime}$ be an integer with $r^{\prime} \geq r$, set $n^{\prime}:=n+\left(r^{\prime}-r\right)$ and let $S^{\prime}=\left\{v_{0}^{\prime}, \ldots, v_{n^{\prime}}^{\prime}\right\}$ be an ordered set of places of $k$ such that $v_{i}^{\prime}=v_{i}$ if $0 \leq i \leq r ; v_{r^{\prime}+i}^{\prime}=v_{r+i}$ if $1 \leq i \leq n-r$; and $\left\{v_{j}^{\prime}: r<j \leq r^{\prime}\right\}$ is a set of $r^{\prime}-r$ places which do not belong to $S$ and are each totally split in $K$. By Čebotarev's density theorem, there exists such an $S^{\prime}$ with the property that $p \nmid \# A_{K, S^{\prime}}$. We may now apply Lemma 2.6 with $S^{\prime}$ and $r^{\prime}$ instead of $S$ and $r$, and with $g_{j}=\sigma$ for each index $j$ with $j>r^{\prime}$. We set $\varepsilon^{\prime}:=x \cdot \bigwedge_{i=1}^{i=r^{\prime}} \epsilon_{i} \in \bigwedge_{\mathbb{Z}[G]}^{r^{\prime}} \mathcal{O}_{K, S^{\prime}}^{\times} \otimes \mathbb{Z}_{(p)}$. Then Proposition 2.7 implies that $\eta_{K, S^{\prime}}$ is equal to the image of $(\sigma-1)^{n-r}\left(\varepsilon^{\prime}\right)$ in $\overline{\bigwedge_{\mathbb{Z}[G]}^{r^{\prime}} \mathcal{O}_{K, S^{\prime}}^{\times}} \otimes \mathbb{Z}_{(p)}$. Now, [24, Proposition 5.2] shows that there is a $\mathbb{Z}[G]$-module 
homomorphism $\Phi=\Phi_{K, S, S^{\prime}}: \overline{\bigwedge_{\mathbb{Z}[G]}^{r^{\prime}} \mathcal{O}_{K, S^{\prime}}^{\times}} \rightarrow \overline{\bigwedge_{\mathbb{Z}[G]}^{r} \mathcal{O}_{K, S}^{\times}}$such that $\eta_{K, S}$ is equal to $\Phi\left(\eta_{K, S^{\prime}}\right)$. Hence we can take $\varepsilon$ to be the image under $\Phi$ of the image of $\varepsilon^{\prime}$ in $\overline{\bigwedge_{\mathbb{Z}[G]}^{r^{\prime}} \mathcal{O}_{K, S^{\prime}}^{\times}} \otimes \mathbb{Z}_{(p)}$, and this satisfies the equality of claim (i).

We next show claim (ii). Proposition 2.7 gives the formulas

$$
\eta_{K, S^{\prime}}=x(\sigma-1)^{n^{\prime}-r^{\prime}} \cdot \bigwedge_{i=1}^{i=r^{\prime}} \epsilon_{i} ; \quad \eta_{k, S^{\prime}}=x\left(\bigwedge_{i=1}^{i=r^{\prime}} N_{K / k} \epsilon_{i}\right) \wedge\left(\bigwedge_{i=r^{\prime}+1}^{i=n^{\prime}} \epsilon_{i}\right) .
$$

Therefore, letting $f_{j}^{\prime}$ correspond to $v_{j}^{\prime}$ as $f_{j}$ does to $v_{j}$, we have

$$
\left(f_{r^{\prime}+1}^{\prime} \wedge \cdots \wedge f_{n^{\prime}}^{\prime}\right)\left(\eta_{k, S^{\prime}}\right)=x \operatorname{det}\left(\left(f_{j}^{\prime}\left(\epsilon_{i}\right)\right)_{r^{\prime}<i, j \leq n^{\prime}}\right) \cdot\left(\bigwedge_{i=1}^{i=r^{\prime}} N_{K / k} \epsilon_{i}\right),
$$

since the $f_{j}^{\prime}$ are trivial on the image of $N_{K / k}$. By Lemma 2.6 (ii), the displayed determinant is that of the identity matrix, and so we obtain

$$
\left(\bigwedge_{\mathbb{Z}[G]}^{r^{\prime}} N_{K / k}\right)\left(\varepsilon^{\prime}\right) \equiv\left(f_{r^{\prime}+1}^{\prime} \wedge \cdots \wedge f_{n^{\prime}}^{\prime}\right)\left(\eta_{k, S^{\prime}}\right)
$$

in $\overline{\bigwedge_{\mathbb{Z}}^{r^{\prime}} \mathcal{O}_{k, S^{\prime}}^{\times}} \otimes \mathbb{Z}_{(p)}$ modulo $\ell$. We have $\eta_{k, S}=\Phi_{k, S, S^{\prime}}\left(\eta_{k, S^{\prime}}\right)$, and so we apply $\Phi_{k, S, S^{\prime}}$ to both sides of this congruence to obtain

$$
\Phi_{k, S, S^{\prime}}\left(\left(\bigwedge_{\mathbb{Z}[G]}^{r^{\prime}} N_{K / k}\right)\left(\varepsilon^{\prime}\right)\right) \equiv\left(f_{r^{\prime}+1}^{\prime} \wedge \cdots \wedge f_{n^{\prime}}^{\prime}\right)\left(\eta_{k, S}\right)
$$

But the definition $[24,(14)]$ shows that $\Phi_{k, S, S^{\prime}} \circ\left(\bigwedge_{\mathbb{Z}[G]}^{r^{\prime}} N_{K / k}\right)=\left(\bigwedge_{\mathbb{Z}[G]}^{r} N_{K / k}\right) \circ$ $\Phi_{K, S, S^{\prime}}$, and therefore we obtain the congruence of claim (ii).

Regarding claim (iii) we first observe that if $k$ is a number field, then the condition $p \nmid \# k_{\text {tors }}^{\times}$implies that $K / k$ has odd degree and hence that all archimedean places belong to $S_{1}$, as claimed. We next describe the definition of the module $\&$. As a $\mathbb{Q}[G]$ module, we have $\mathbb{Q} \mathcal{O}_{K, S}^{\times} \cong \mathbb{Q}[G]^{r} \oplus \mathbb{Q}^{n-r}$. Hence, considering the component away from the trivial character, we have $\bigwedge_{\mathbb{Q}[G] e}^{r} e \mathbb{Q} \mathcal{O}_{K, S}^{\times}=\mathbb{Q}[G] \cdot e \varepsilon$, since $e_{\chi} \varepsilon \neq 0$ for all nontrivial $\chi$ (by its relation to the values of $L$-functions). Now the $\mathbb{Q}[G] e$-module $e \mathbb{Q} \mathcal{O}_{K, S}^{\times}$is isomorphic to $(\mathbb{Q}[G] e)^{r}$ and $\bigwedge_{\mathbb{Q}[G] e}^{r}(\mathbb{Q}[G] e)^{r}$ is a cyclic $\mathbb{Q}[G] e$-module generated by the primitive tensor corresponding to the standard basis of $(\mathbb{Q}[G] e)^{r}$. We may therefore conclude that $e \varepsilon$ may be written as a primitive tensor

$$
e \varepsilon=q \cdot e \alpha_{1} \wedge e \alpha_{2} \wedge \cdots \wedge e \alpha_{r},
$$

with $q^{-1} \in \mathbb{Z} \backslash\{0\}$ and each $\alpha_{i}$ an element of $\mathcal{O}_{K, S}^{\times}$. For any nontrivial character $\chi$, we therefore have

$$
e_{\chi} \cdot \alpha_{1} \wedge \alpha_{2} \wedge \cdots \wedge \alpha_{r} \neq 0
$$

But expression (6) is not changed by multiplying any $\alpha_{i}$ by an element of $\mathcal{O}_{k, S}^{\times}$, and by doing so one may ensure that (7) holds for $\chi$ the trivial character as well. If we then take $\mathcal{E}$ to be the $G$-submodule of $\mathcal{O}_{K, S}^{\times}$that is generated by $\left\{\alpha_{i}: 1 \leq i \leq r\right\}$, 
this module is free of rank $r$ and the equality $\bigwedge_{\mathbb{Z}[G]}^{r} \& \otimes \mathcal{A}_{(p)}=t_{\mathcal{E}} \mathcal{A}_{(p)} \varepsilon$ holds with $t_{\mathscr{E}}$ equal to the maximal power of $p$ which divides $q^{-1}$.

If we now assume that $r=1$, then we may simply take $\varepsilon=\mathbb{Z}_{(p)}[G] \varepsilon$, and this is free of rank 1 (adjusting $\varepsilon$ by an element of $\mathcal{O}_{k, S}^{\times}$if necessary, which does not affect the validity of either claim (i) or claim (ii)). Therefore we may take $t_{\mathcal{E}}=1$ in the case $r=1$.

We have now proved all of Theorem 3.1 apart from the inclusions of claim (iii). In the rest of this section we deduce these via homological algebra from Conjecture $\mathrm{C}(K / k)$.

It is straightforward to verify that there exists a free $\mathbb{Z}[G]$-submodule $\mathcal{F}$ of $\Psi_{S}^{1}$ which is of rank $r$ and projects to $\sum_{i=1}^{i=r} \mathbb{Z}[G] \cdot\left(w_{i}-w_{0}\right)$ under the composite surjection $\Psi_{S}^{1} \rightarrow X_{K, S}^{\prime} \rightarrow X_{K, S}$, where the first and second arrows are as in (1) and (2) respectively. We use the same symbol to denote the image of $\mathcal{F}$ in $X_{K, S}^{\prime}$. We also let $F^{\bullet}$ denote the complex $\mathscr{E} \stackrel{0}{\rightarrow} \mathcal{F}$, where the first term occurs in degree 0 . Then one has a tautological short exact sequence of perfect complexes

$$
0 \longrightarrow F^{\bullet} \longrightarrow \Psi_{S}^{\bullet} \longrightarrow \Phi_{S}^{\bullet} \longrightarrow 0
$$

where the second arrow denotes the natural 'inclusion' map and $\Phi_{S}^{\bullet}$ denotes the complex obtained from the middle two terms in the following exact sequence (induced by (1))

$$
0 \longrightarrow \mathcal{O}_{K, S}^{\times} / \mathscr{E} \longrightarrow \Psi_{S}^{0} / \mathscr{E} \longrightarrow \Psi_{S}^{1} / \mathcal{F} \longrightarrow X_{K, S}^{\prime} / \mathcal{F} \longrightarrow 0 .
$$

We must now extend scalars from $\mathbb{Z}[G]$ to $\mathcal{A}$. For any two $G$-modules $M$ and $N$, the 'diagonal' $G$-action on $M \otimes N$ is the action by which each $g \in G$ sends $m \otimes n \in M \otimes N$ to $g^{-1} m \otimes g n$. In the special case that $N$ is equal to $\mathbb{Z}[G]$, the resulting module is isomorphic to $M \otimes \mathbb{Z}[G]$, where $G$ acts only on the second factor (in the canonical way). The isomorphism from the former to the latter is given by taking $m \otimes g$ to $g m \otimes g$. It follows that for any finitely-generated free $\mathbb{Z}[G]$-module $F$, isomorphic modules are obtained from $M \otimes F$ by taking either the diagonal action, or the action on the second factor.

We now apply the exact functor $\mathcal{A} \otimes_{\mathbb{Z}}-$ to (9), endow the resulting groups with the diagonal $G$-action, and compare taking $G$-invariants and $G$-coinvariants. This gives the following commutative diagram:

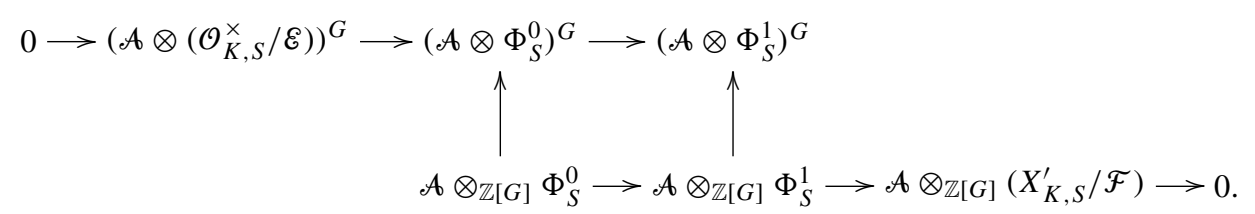


The vertical maps here are those which are induced by the action of $\mathrm{T}$ and are bijective since the $G$-modules $\Phi_{S}^{0}$ and $\Phi_{S}^{1}$, and hence also $\mathcal{A} \otimes \Phi_{S}^{0}$ and $\mathcal{A} \otimes \Phi_{S}^{1}$, are cohomologically trivial. Hence one has an exact sequence of $\mathcal{A}$-modules

$$
\begin{aligned}
0 \longrightarrow & \left(\mathcal{A} \otimes\left(\mathcal{O}_{K, S}^{\times} / \mathscr{E}\right)\right)^{G} \longrightarrow \mathcal{A} \otimes_{\mathbb{Z}[G]} \Phi_{S}^{0} \\
& \longrightarrow \mathcal{A} \otimes_{\mathbb{Z}[G]} \Phi_{S}^{1} \longrightarrow \mathcal{A} \otimes_{\mathbb{Z}[G]}\left(X_{K, S}^{\prime} / \mathcal{F}\right) \longrightarrow 0 .
\end{aligned}
$$

Now $G$ acts trivially on both $\mathbb{Q} \otimes \mathcal{O}_{K, S}^{\times} / \mathscr{E}$ and $\mathbb{Q} \otimes X_{K, S}^{\prime} / \mathcal{F}$, and so the second and fifth terms in the above sequence are finite. This observation implies that there is a canonical isomorphism

$$
\iota: \mathbb{Q}[G] e \otimes_{\mathbb{Z}[G]} \operatorname{Det}_{\mathbb{Z}[G]}\left(\Phi_{S}^{\bullet}\right) \cong(\mathbb{Q}[G] e, 0) .
$$

Furthermore, the approach of [12, proof of Corollary 2] allows one to compute $\iota\left(\operatorname{Det}_{\mathcal{A}_{(p)}}\left(\mathcal{A}_{(p)} \otimes_{\mathbb{Z}[G]} \Phi_{S}^{\bullet}\right)\right)$. Indeed, after observing that $\mathcal{A}_{(p)}$ satisfies the conditions on $R$ in [loc. cit., Lemma 5], we may deduce from the exactness of (10) an equality of the form

$$
\begin{gathered}
\operatorname{Fitt}_{\mathcal{A}_{(p)}}\left(\left(\mathcal{A}_{(p)} \otimes\left(\mathcal{O}_{K, S}^{\times} / \mathcal{E}\right)\right)^{G}\right) \operatorname{Det}_{\mathcal{A}_{(p)}}\left(\mathcal{A}_{(p)} \otimes_{\mathbb{Z}[G]} \Phi_{S}^{\bullet}\right) \\
=\operatorname{Fitt}_{\mathcal{A}_{(p)}}\left(\mathcal{A}_{(p)} \otimes_{\mathbb{Z}[G]}\left(X_{K, S}^{\prime} / \mathcal{F}\right)\right) .
\end{gathered}
$$

(Here, when applying [loc. cit., Lemma 5], we have also used the fact that, since $G$ is cyclic, the argument of [22, Appendix, Proposition 1] implies the equality $\operatorname{Fitt}_{\mathcal{A}_{(p)}}\left(\operatorname{Hom}_{\mathbb{Z}_{(p)}}\left(M, \mathbb{Q} / \mathbb{Z}_{(p)}\right)\right)=\operatorname{Fitt}_{\mathcal{A}_{(p)}}(M)$ for any finite $\mathcal{A}_{(p)}$-module $\left.M.\right)$

In the next three results we provide explicit information on each of the terms in (11).

Lemma 3.5. If Conjecture $\mathrm{C}(K / k)$ is valid, then one has

$$
\operatorname{Det}_{\mathcal{A}_{(p)}}\left(\mathcal{A}_{(p)} \otimes_{\mathbb{Z}[G]} \Phi_{S}^{\bullet}\right)=\mathcal{A}_{(p)} \cdot t_{\mathcal{E}}^{-1}(\sigma-1)^{n-r} .
$$

Proof. Since $\mathcal{A}_{(p)} \otimes_{\mathbb{Z}[G]} \mathcal{E}$ and $\mathcal{A}_{(p)} \otimes_{\mathbb{Z}[G]} \mathcal{F}$ are both $\mathbb{Z}_{(p)}$-torsion-free, whilst the groups $\operatorname{Tor}_{\mathbb{Z}[G]}^{1}\left(\mathcal{A}_{(p)}, \Phi_{S}^{0}\right)$ and $\operatorname{Tor}_{\mathbb{Z}[G]}^{1}\left(\mathcal{A}_{(p)}, \Phi_{S}^{1}\right)$ are both finite, (8) induces an exact sequence of complexes of $\mathcal{A}_{(p)}$-modules

$$
0 \rightarrow \mathcal{A}_{(p)} \otimes_{\mathbb{Z}[G]} F^{\bullet} \rightarrow \mathcal{A}_{(p)} \otimes_{\mathbb{Z}[G]} \Psi_{S}^{\bullet} \rightarrow \mathcal{A}_{(p)} \otimes_{\mathbb{Z}[G]} \Phi_{S}^{\bullet} \rightarrow 0,
$$

and hence also an isomorphism in $\mathcal{P}\left(\mathcal{A}_{(p)}\right)$ of the form

$\operatorname{Det}_{\mathcal{A}_{(p)}}\left(\mathcal{A}_{(p)} \otimes_{\mathbb{Z}[G]} F^{\bullet}\right) \otimes_{\mathcal{P}\left(\mathcal{A}_{(p)}\right)} \operatorname{Det}_{\mathcal{A}_{(p)}}\left(\mathcal{A}_{(p)} \otimes_{\mathbb{Z}[G]} \Phi_{S}^{\bullet}\right) \cong \operatorname{Det}_{\mathcal{A}_{(p)}}\left(\mathcal{A}_{(p)} \otimes_{\mathbb{Z}[G]} \Psi_{S}^{\bullet}\right)$.

Now if $C^{\bullet}$ denotes any of the complexes $F^{\bullet}, \Psi_{S}^{\bullet}$ or $\Phi_{S}^{\bullet}$, then $\mathcal{A}_{(p)} \otimes_{\mathbb{Z}[G]} C^{\bullet}$ is naturally isomorphic to $\mathcal{A}_{(p)} \otimes_{\mathbb{Z}[G]}^{\mathbb{L}} C^{\bullet}$ and so $\operatorname{Det}_{\mathcal{A}_{(p)}}\left(\mathcal{A}_{(p)} \otimes_{\mathbb{Z}[G]} C^{\bullet}\right)$ identifies with $\mathcal{A}_{(p)} \otimes_{\mathbb{Z}[G]} \operatorname{Det}_{\mathbb{Z}[G]} C^{\bullet}$. 
In particular, therefore, the validity of Conjecture $\mathrm{C}(K / k)$ would imply that

$$
\left(\mathbb{R}[G] e \otimes_{\mathbb{R}[G]} \vartheta_{\Psi_{S}, \mathbb{R}}\right)\left(\operatorname{Det}_{\mathcal{A}_{(p)}}\left(\mathcal{A}_{(p)} \otimes_{\mathbb{Z}[G]} \Psi_{S}^{\bullet}\right)\right)=\left(\mathcal{A}_{(p)} \cdot \theta_{K / k, S}^{r}(0), 0\right) .
$$

We combine (12) with the isomorphism $\iota$ to identify $\operatorname{Det}_{\mathbb{Q}[G] e}\left(\mathbb{Q}[G] e \otimes_{\mathbb{Z}[G]} F^{\bullet}\right)$ with $\operatorname{Det}_{\mathbb{Q}[G] e}\left(\mathbb{Q}[G] e \otimes_{\mathbb{Z}[G]} \Psi_{S}^{\bullet}\right.$ ). Then, in view of (12) and the last displayed equality, the claimed result is equivalent to an equality

$\left(\mathbb{R}[G] e \otimes_{\mathbb{R}[G]} \vartheta_{\Psi_{S}, \mathbb{R}}\right)\left(\operatorname{Det}_{\mathcal{A}_{(p)}}\left(\mathcal{A}_{(p)} \otimes_{\mathbb{Z}[G]} F^{\bullet}\right)\right)=\left(\mathcal{A}_{(p)} \cdot t_{\mathcal{E}}(\sigma-1)^{r-n} \theta_{K / k, S}^{r}(0), 0\right)$.

But

$\operatorname{Det}_{\mathcal{A}_{(p)}}\left(\mathcal{A}_{(p)} \otimes_{\mathbb{Z}[G]} F^{\bullet}\right)=\operatorname{Det}_{\mathcal{A}_{(p)}}\left(\mathcal{A}_{(p)} \otimes_{\mathbb{Z}[G]} \mathcal{E}\right) \otimes_{\mathcal{P}\left(\mathcal{A}_{(p)}\right)} \operatorname{Det}_{\mathcal{A}_{(p)}}\left(\mathcal{A}_{(p)} \otimes_{\mathbb{Z}[G]} \mathcal{F}\right)^{-1}$

Also, if we pick a basis $\alpha_{1}, \ldots, \alpha_{r}$ for the free module $\mathcal{E}$, then by what we have proved so far of Theorem 3.1 (iii), there exists an element $u$ of $\mathbb{Z}_{(p)}[G]^{\times}$such that $e \bigwedge_{i=1}^{i=r} \alpha_{i}=e u t_{\mathcal{E}}(\varepsilon)=u t_{\mathcal{E}}(\sigma-1)^{r-n} e\left(\eta_{K, S}\right)$. Therefore

$$
\begin{aligned}
e\left(\bigwedge_{\mathbb{R}[G]}^{r} \operatorname{Reg}_{K, S}\right)\left(\bigwedge_{i=1}^{i=r} \alpha_{i}\right) & =u t_{\mathcal{E}}(\sigma-1)^{r-n} e\left(\bigwedge_{\mathbb{R}[G]}^{r} \operatorname{Reg}_{K, S}\right)\left(\eta_{K, S}\right) \\
& =u t_{\mathcal{E}}(\sigma-1)^{r-n} e \theta_{K / k, S}^{r}(0) \bigwedge_{i=1}^{i=r}\left(w_{i}-w_{0}\right),
\end{aligned}
$$

the last equality following from the definition (4) of $\eta_{K, S}$. Hence the image of (14) under $\mathbb{R}[G] e \otimes_{\mathbb{R}[G]} \vartheta_{\Psi_{S}^{\bullet}, \mathbb{R}}$ is equal to $\left(\mathcal{A}_{(p)} \cdot u t_{\mathcal{E}}(\sigma-1)^{r-n} \theta_{K / k, S}^{r}(0), 0\right)$, and this implies the required equality (13) because $\mathcal{A}_{(p)} \cdot u=\mathcal{A}_{(p)}$.

Lemma 3.6. Fitt $_{\mathcal{A}}\left(\mathcal{A} \otimes_{\mathbb{Z}[G]}\left(X_{K, S}^{\prime} / \mathcal{F}\right)\right)=(\sigma-1)^{n-r} \operatorname{Fitt}_{\mathbb{Z}[G]}\left(A_{K, S}\right) \mathcal{A}$.

Proof. We have the exact sequence

$$
0 \longrightarrow A_{K, S} \longrightarrow X_{K, S}^{\prime} / \mathcal{F} \longrightarrow \sum_{i=r+1}^{i=n} \mathbb{Z}[G] \cdot\left(w_{i}-w_{0}\right) \longrightarrow 0
$$

where the last nonzero term is isomorphic as a $\mathbb{Z}[G]$-module to $\mathbb{Z}^{n-r}$. Applying $\mathcal{A} \otimes_{\mathbb{Z}[G]}$ - therefore gives an exact sequence

$$
0 \longrightarrow \mathcal{A} \otimes_{\mathbb{Z}[G]} A_{K, S} \longrightarrow \mathcal{A} \otimes_{\mathbb{Z}[G]}\left(X_{K, S}^{\prime} / \mathcal{F}\right) \longrightarrow\left(\mathcal{A} \otimes_{\mathbb{Z}[G]} \mathbb{Z}\right)^{n-r} \longrightarrow 0 .
$$

(Here we use the fact that $\operatorname{Tor}_{\mathbb{Z}[G]}^{1}(\mathcal{A}, \mathbb{Z})=0$, as can be easily verified directly.) In addition, the exact sequence $0 \rightarrow \mathcal{A} \stackrel{1 \mapsto \sigma-1}{\longrightarrow} \mathcal{A} \rightarrow \mathcal{A} \otimes_{\mathbb{Z}[G]} \mathbb{Z} \rightarrow 0$ shows that [13, Lemma 3] applies to (15) and therefore implies that

$\operatorname{Fitt}_{\mathcal{A}}\left(\mathcal{A} \otimes_{\mathbb{Z}[G]}\left(X_{K, S}^{\prime} / \mathcal{F}\right)\right)=\operatorname{Fitt}_{\mathcal{A}}\left(\mathcal{A} \otimes_{\mathbb{Z}[G]} A_{K, S}\right) \operatorname{Fitt}_{\mathcal{A}}\left(\left(\mathcal{A} \otimes_{\mathbb{Z}[G]} \mathbb{Z}\right)^{n-r}\right)$.

But Fitt $\mathcal{A}_{\mathcal{A}}\left(\mathcal{A} \otimes_{\mathbb{Z}[G]} A_{K, S}\right)=\operatorname{Fitt}_{\mathbb{Z}[G]}\left(A_{K, S}\right) \mathcal{A}$ and $\operatorname{Fitt}_{\mathcal{A}}\left(\left(\mathcal{A} \otimes_{\mathbb{Z}[G]} \mathbb{Z}\right)^{n-r}\right)=$ $\mathcal{A}(\sigma-1)^{n-r}$, and this implies the claimed result. 
By first substituting the last two results into (11), and then cancelling the element $(\sigma-1)^{n-r}$ (which is a unit in $\mathbb{Q}[G] e$ ) from the resulting equalities, we obtain

$$
t_{\mathcal{E}}^{-1} \operatorname{Fitt}_{\mathcal{A}_{(p)}}\left(\left(\mathcal{A}_{(p)} \otimes\left(\mathcal{O}_{K, S}^{\times} / \mathcal{E}\right)\right)^{G}\right)=\operatorname{Fitt}_{\mathbb{Z}[G]}\left(A_{K, S}\right) \mathcal{A}_{(p)} .
$$

Our proof of Theorem 3.1 will therefore be completed by the following lemma.

Lemma 3.7. Let I denote the group of fractional ideals supported above $S \backslash S_{1}$, and $\langle\mathcal{E}\rangle$ the image of $\mathcal{E}$ under the natural map $\mathcal{O}_{K, S}^{\times} \rightarrow I$. Then $\langle\mathcal{E}\rangle$ is a free $\mathbb{Z}$-module of rank $b$ with $b \leq r$, and one has

$$
\begin{aligned}
(\sigma-1)^{b} \cdot \operatorname{Fitt}_{\mathcal{A}}\left(\left(\mathcal{O}_{K, S_{1}}^{\times} / \mathcal{O}_{K, S_{1}}^{\times} \cap \mathcal{E}\right)^{\mathrm{T}=0}\right) & \subseteq \operatorname{Fitt}_{\mathcal{A}}\left(\left(\mathcal{A} \otimes\left(\mathcal{O}_{K, S}^{\times} / \mathscr{E}\right)\right)^{G}\right) \\
& \subseteq \operatorname{Fitt}_{\mathcal{A}}\left(\left(\mathcal{O}_{K, S_{1}}^{\times} / \mathcal{O}_{K, S_{1}}^{\times} \cap \mathcal{E}\right)^{\mathrm{T}=0}\right) .
\end{aligned}
$$

Further, the image of the natural map $\left(\mathcal{A} \otimes\left(\mathcal{O}_{K, S}^{\times} / \mathscr{E}\right)\right)^{G} \rightarrow(\mathcal{A} \otimes(I /\langle\mathscr{E}\rangle))^{G}$ is finite of order dividing $\ell^{b}$, and the second, respectively first, displayed inclusion is an equality if the image of this map is trivial, respectively has order $\ell^{b}$.

Proof. If $M$ is any $G$-module, then by applying $-\otimes_{\mathbb{Z}} M$ to the augmentation sequence $0 \rightarrow \mathcal{A} \stackrel{1 \mapsto \sigma-1}{\longrightarrow} \mathbb{Z}[G] \rightarrow \mathbb{Z} \rightarrow 0$ and then taking fixed points under the diagonal $G$ action one obtains an exact sequence of $G$-modules of the form $0 \rightarrow\left(\mathcal{A} \otimes_{\mathbb{Z}} M\right)^{G} \rightarrow$ $M \stackrel{\mathrm{T}}{\rightarrow} M^{G}$ (where the $G$-action on the term $\left(\mathcal{A} \otimes_{\mathbb{Z}} M\right)^{G}$ is by multiplication on the first factor).

By applying this observation to each of the modules in the natural exact sequence

$$
0 \rightarrow \mathcal{O}_{K, S_{1}}^{\times} / \mathcal{O}_{K, S_{1}}^{\times} \cap \mathcal{E} \rightarrow \mathcal{O}_{K, S}^{\times} / \mathscr{E} \rightarrow I /\langle\mathcal{E}\rangle
$$

we obtain an exact commutative diagram

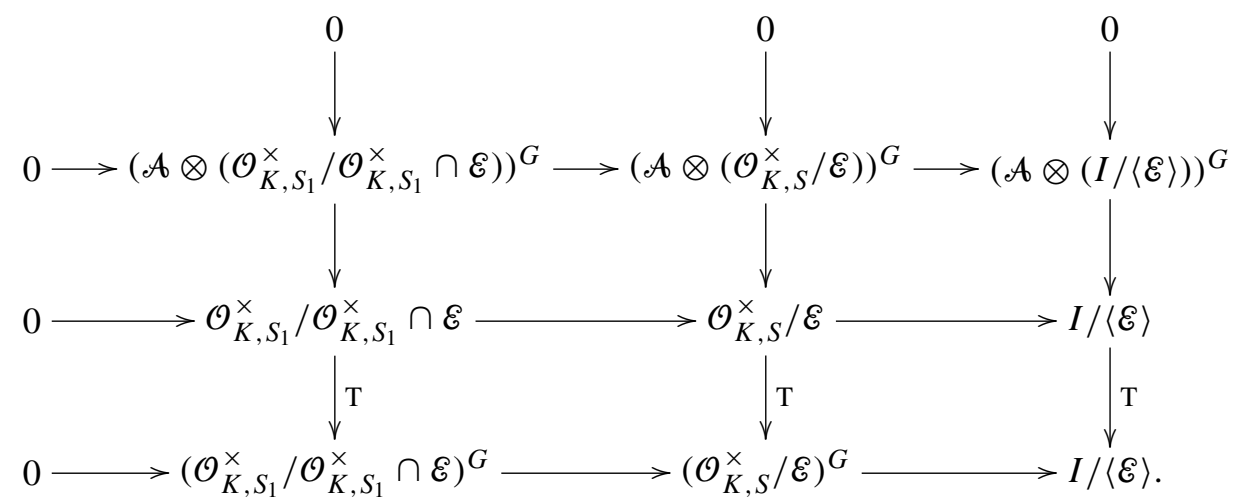

Now $G$ acts trivially on the free $\mathbb{Z}$-module $I$ so that $\operatorname{ker}(I /\langle\mathscr{E}\rangle \stackrel{\mathrm{T}}{\rightarrow} I /\langle\mathscr{E}\rangle)$ is isomorphic to a submodule of $\operatorname{cok}(\langle\mathscr{E}\rangle \stackrel{\cdot \ell}{\rightarrow}\langle\mathcal{E}\rangle) \cong(\mathbb{Z} / \ell \mathbb{Z})^{b}$ where $b$ is the rank of the free $\mathbb{Z}$ module $\langle\mathscr{E}\rangle$. (We also note that, since $\langle\mathscr{E}\rangle$ is a quotient of $H_{0}(G, \mathcal{E}) \cong \mathbb{Z}^{r}$ one has 
$b \leq r$, as claimed.) The above diagram thus gives rise to an exact sequence of $\mathcal{A}$-modules of the form

$$
0 \longrightarrow\left(\mathcal{O}_{K, S_{1}}^{\times} / \mathcal{O}_{K, S_{1}}^{\times} \cap \mathcal{E}\right)^{\mathrm{T}=0} \rightarrow\left(\mathcal{A} \otimes\left(\mathcal{O}_{K, S}^{\times} / \mathscr{E}\right)\right)^{G} \rightarrow(\mathbb{Z} / \ell \mathbb{Z})^{b} .
$$

But $\mathbb{Z} / \ell \mathbb{Z} \cong \mathscr{A} / \mathcal{A}(\sigma-1)$ so that the exact sequence

$$
0 \longrightarrow \mathcal{A} \stackrel{1 \mapsto \sigma-1}{\longrightarrow} \mathcal{A} \longrightarrow \mathbb{Z} / \ell \mathbb{Z} \longrightarrow 0
$$

implies Fitt $_{\mathcal{A}}\left((\mathbb{Z} / \ell \mathbb{Z})^{b}\right)=\left(\operatorname{Fitt}_{\mathcal{A}}(\mathbb{Z} / \ell \mathbb{Z})\right)^{b}=\mathcal{A}(\sigma-1)^{b}$ and, since $G$ is cyclic, Fitt $_{\mathcal{A}}(N) \subseteq$ Fitt $_{\mathcal{A}}(M)$ for any finite $\mathcal{A}$-modules $M \subseteq N$. Hence, the exact sequence (16) combines with well-known properties of Fitting ideals with respect to short exact sequences (cf. [21, Chapter XIX, Proposition 2.7]) to imply that

$$
\begin{aligned}
(\sigma-1)^{b} \cdot \operatorname{Fitt}_{\mathcal{A}}\left(\left(\mathcal{O}_{K, S_{1}}^{\times} / \mathcal{O}_{K, S_{1}}^{\times} \cap \mathcal{E}\right)^{\mathrm{T}=0}\right) & \subseteq \operatorname{Fitt}_{\mathcal{A}}\left(\left(\mathcal{A} \otimes\left(\mathcal{O}_{K, S}^{\times} / \mathscr{E}\right)\right)^{G}\right) \\
& \subseteq \operatorname{Fitt}_{\mathcal{A}}\left(\left(\mathcal{O}_{K, S_{1}}^{\times} / \mathcal{O}_{K, S_{1}}^{\times} \cap \mathcal{E}\right)^{\mathrm{T}=0}\right),
\end{aligned}
$$

as claimed.

Finally, we observe that the image $B$ of the map

$$
\left(\mathcal{A} \otimes\left(\mathcal{O}_{K, S}^{\times} / \mathscr{E}\right)\right)^{G} \longrightarrow(\mathcal{A} \otimes(I /\langle\mathscr{E}\rangle))^{G}
$$

is isomorphic to a submodule of $(\mathbb{Z} / \ell \mathbb{Z})^{b}$ and hence has order dividing $\ell^{b}$. Now if $B$ is trivial, then (16) reduces to give an isomorphism between $\left(\mathcal{O}_{K, S_{1}}^{\times} / \mathcal{O}_{K, S_{1}}^{\times} \cap \mathcal{E}\right)^{\mathrm{T}=0}$ and $\left(\mathcal{A} \otimes\left(\mathcal{O}_{K, S}^{\times} / \mathscr{E}\right)\right)^{G}$ so that the second displayed inclusion is an equality. On the other hand, if $B$ has order $\ell^{b}$, then (16) is a short exact sequence and by applying [13, Lemma 3] to this sequence (which is permissible by virtue of (17)) we find that the first displayed inclusion is an equality.

We now finish this section by providing a condition under which the image of the map mentioned in the statement of Lemma 3.7 is trivial.

Lemma 3.8. Let $P$ denote the image of $\mathcal{O}_{K, S}^{\times}$in $I$. For any group $A$, let $A^{\times \ell}$ denote the set of $\ell$-th powers in $A$. If $P^{\times \ell} \cap\langle\mathcal{E}\rangle=\langle\mathscr{E}\rangle^{\times \ell}$, then

$$
\operatorname{Fitt}_{\mathcal{A}}\left(\left(\mathcal{A} \otimes\left(\mathcal{O}_{K, S}^{\times} / \mathscr{E}\right)\right)^{G}\right)=\operatorname{Fitt}_{\mathcal{A}}\left(\left(\mathcal{O}_{K, S_{1}}^{\times} / \mathcal{O}_{K, S_{1}}^{\times} \cap \mathcal{E}\right)^{\mathrm{T}=0}\right) .
$$

Proof. Applying the snake lemma to the exact diagram

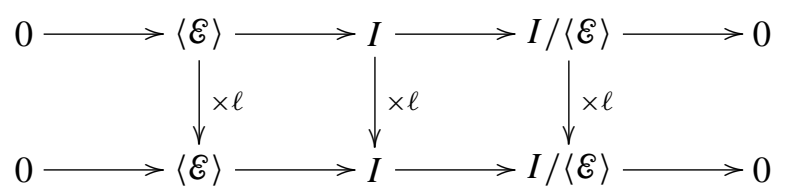

yields an inclusion $(\mathcal{A} \otimes(I /\langle\mathscr{E}\rangle))^{G} \rightarrow\langle\mathcal{E}\rangle /\langle\mathscr{E}\rangle^{\times \ell}$, under which the image of the map described in the statement of Lemma 3.7 is contained in $\left(P^{\times \ell} \cap\langle\mathcal{E}\rangle\right) /\langle\mathscr{E}\rangle^{\times \ell}$. 


\section{Further examples}

The following result shows that if $\operatorname{Gal}(K / \mathbb{Q})$ is not cyclic, then there is no direct analogue of Corollary 3.2.

Proposition 4.1. There exists an infinite family of non-cyclic finite abelian extensions $K / \mathbb{Q}$ in which every ramified prime has decomposition subgroup equal to $G:=$ $\mathrm{Gal}(K / \mathbb{Q})$, and yet the conductor-level Sinnott unit of $K$ cannot be expressed in the form $\prod_{i} \lambda_{i}\left(u_{i}\right)$ with each $u_{i}$ an element of $\mathcal{O}_{K, S}^{\times}$and each $\lambda_{i}$ an element of the augmentation ideal $I_{G}$ of $\mathbb{Z}[G]$, where $S$ is the set of places of $\mathbb{Q}$ which are either archimedean or ramified in $K$.

Proof. Fix an odd prime $p$ and let $p_{1}, p_{2}$ be distinct rational primes congruent to 1 modulo $p$. For $i=1,2$, assume that $p_{i}$ is not a $p$-th power modulo $p_{3-i}$, and let $K_{i}$ be an extension of $\mathbb{Q}$ of degree $p$ and conductor $p_{i}^{a_{i}}$ for some integer $a_{i}>0$. Let $K$ be the compositum $K_{1} K_{2}$, so $K / \mathbb{Q}$ has Galois group $G \cong(\mathbb{Z} / p \mathbb{Z})^{2}$, each $p_{i}$ has decomposition subgroup equal to $G$, and we may take $S=\left\{\infty, p_{1}, p_{2}\right\}$. We recall that such fields have been studied in detail by Fröhlich and, in particular, that it was proved in [15] that the class number of $K$ is coprime to $p$.

As in $\S 3$, for any $\mathbb{Z}[G]$-module $A$ we set $\bar{A}:=A / A_{\text {tors }}$. We also let $A^{\mathrm{T}=0}$, resp. $A^{I_{G}}$, denote the kernel of the endomorphism of $A$ that is induced by the action of the element $\sum_{g \in G} g \in \mathbb{Z}[G]$, resp. the submodule of $A^{\mathrm{T}=0}$ that is generated by all elements of the form $i(a)$ with $a \in A$ and $i \in I_{G}$.

Lemma 4.2. ${\overline{\mathcal{O}_{K, S}^{\times}}}^{\mathrm{T}}=0{\overline{\mathcal{O}_{K, S}^{\times}}}^{I_{G}}=H^{-1}\left(G, \overline{\mathcal{O}_{K, S}^{\times}}\right) \neq 0$.

Proof. The first equality follows directly from the definition of $H^{-1}\left(G, \overline{\mathcal{O}_{K, S}^{\times}}\right)$, and so we need only show that $H^{-1}\left(G, \overline{\mathcal{O}_{K, S}^{\times}}\right)$does not vanish.

First note that $\left(\mathcal{O}_{K, S}^{\times}\right)$tors $=\{ \pm 1\}$ is a cohomologically trivial $G$-module (since $\# G$ is odd) and hence that $H^{-1}\left(G, \overline{\mathcal{O}_{K, S}^{\times}}\right)$is canonically isomorphic to $H^{-1}\left(G, \mathcal{O}_{K, S}^{\times}\right)$. Now applying the double coboundary isomorphism induced by the exact sequence (1) (which exists by our definition of $S$ ) shows that $H^{-1}\left(G, \mathcal{O}_{K, S}^{\times}\right)$is isomorphic to $H^{-3}\left(G, X_{K, S}^{\prime}\right)$. In addition, $A_{K, S}$ is a cohomologically trivial $G$-module because $p \nmid \# A_{K, S}$, and so the exact sequence (2) induces an isomorphism $H^{-3}\left(G, X_{K, S}^{\prime}\right) \cong$ $H^{-3}\left(G, X_{K, S}\right)$. But $X_{K, S}$ is isomorphic to $\mathbb{Z}[G] \oplus \mathbb{Z}$ by the definition of $S$, and hence one has an isomorphism $H^{-1}\left(G, \overline{\mathcal{O}_{K, S}^{\times}}\right) \cong H^{-3}(G, \mathbb{Z})$. On the other hand, this last group is $H_{2}(G, \mathbb{Z})$ and is therefore isomorphic to $\bigwedge_{\mathbb{Z}}^{2} G$ [2, Chapter V, Theorem 6.4]. But, in our case, $\bigwedge_{\mathbb{Z}}^{2} G$ is isomorphic to $\mathbb{Z} / p \mathbb{Z}$ and hence $H^{-1}\left(G, \overline{\mathcal{O}_{K, S}^{\times}}\right)$does not vanish, as required. 
We return to the proof of Proposition 4.1. Let $\eta=N_{\mathbb{Q}\left(\zeta_{p_{1}} \zeta_{p_{2}} a_{2}\right) / K}\left(1-\zeta_{p_{1}} \zeta_{p_{2}} \zeta_{a_{2}}\right)$ be the conductor-level Sinnott unit of $K$. Then, since $p$ is odd, the argument of [3, proof of Lemma 6.2] implies that the image of $\eta$ in $\overline{\mathcal{O}_{K, S}^{\times}} \otimes \mathbb{Z}_{p}$ is a $\mathbb{Z}_{p}[G]$-generator of ${\overline{\mathcal{O}_{K, S}^{\times}}}^{\mathrm{T}}=0 \quad \otimes \mathbb{Z}_{p}$. It follows that the image of $\eta$ in $\overline{\mathcal{O}_{K, S}^{\times}}$generates a nontrivial subgroup of $H^{-1}\left(G, \overline{\mathcal{O}_{K, S}^{\times}}\right)$and hence that $\eta$ does not belong to $\left(\mathcal{O}_{K, S}^{\times}\right)^{I_{G}}$, as claimed.

\section{References}

[1] W. Bley and D. Burns, Explicit units and the equivariant Tamagawa number conjecture. Amer. J. Math. 123 (2001), 931-949. Zbl 0984.11055 MR 1854115

[2] K. S. Brown, Cohomology of Groups, Grad. Text in Math. 87, Springer-Verlag, New York 1982. Zbl 0584.20036 MR 0672956

[3] D. Burns, On multiplicative Galois structure invariants. Amer. J. Math. 117 (1995), 875-904. Zbl 0863.11076 MR 1342834

[4] D. Burns, Equivariant Tamagawa Numbers and Galois module theory. Compositio Math. 129 (2001), 203-237. Zbl 1014.11070 MR 1863302

[5] D. Burns, Equivariant Tamagawa Numbers and refined abelian Stark conjectures. J. Math. Soc. Univ. Tokyo. 10 (2003), 225-259. Zbl 1037.11072 MR 1987132

[6] D. Burns, On the values of equivariant Zeta functions on curves over finite fields. Documenta Math. 9 (2004), 357-399. Zbl 1077.11049 MR 2117419

[7] D. Burns, Congruences between derivatives of abelian $L$-functions at $s=0$. Invent. Math., to appear.

[8] D. Burns and M. Flach, On Galois structure invariants associated to Tate motives. Amer. J. Math. 120 (1998), 1343-1397. Zbl 0929.11050 MR 1657186

[9] D. Burns and M. Flach, Tamagawa numbers for motives with (non-commutative) coefficients. Documenta Math. 6 (2001), 501-570. Zbl 1052.11077 MR 1884523

[10] D. Burns and M. Flach, Tamagawa numbers for motives with (noncommutative) coefficients, II. Amer. J. Math. 125 (2003), 475-512. Zbl 01940860 MR 1981031

[11] D. Burns and C. Greither, On the equivariant Tamagawa number conjecture for Tate motives. Invent. Math. 153 (2003), 303-359. Zbl 02001021 MR 1992015

[12] D. Burns and C. Greither, Equivariant Weierstrass preparation and values of $L$-functions at negative integers. Documenta Math., Extra Volume: Kazuya Kato's Fiftieth Birthday (2003), 157-185. Zbl 02028834 MR 2046598

[13] P. Cornacchia and C. Greither, Fitting ideals of class groups of real fields with prime power conductor. J. Number Theory 73 (1998), 459-471. Zbl 0926.11085 MR 1658000

[14] M. Flach, The equivariant Tamagawa number conjecture: a survey (with an Appendix by C. Greither). In Stark's conjectures: recent work and new directions, Contemp. Math. 358, Amer. Math. Soc., Providence, RI, 2004, 79-126. Zbl 1070.11025 MR 2088713 
Vol. 82 (2007) Explicit units and the equivariant Tamagawa number conjecture, II

[15] A. Fröhlich, On the absolute class-group of Abelian fields I; II. J. London Math. Soc. 29 (1954), 211-217; 30 (1955), 72-80. Zbl 0055.03302 MR 0066422 Zbl 0064.27305 MR 0066424

[16] C. Greither and R. Kučera, Annihilators for the class group of a cyclic field of prime power degree. Acta Arith. 12 (2004), 177-198. Zbl 1065.11089 MR 2051376

[17] A. Hayward, A class number formula for higher derivatives of abelian $L$-functions. Compositio Math. 140 (2004), 99-129. Zbl 1060.11075 MR 1984423

[18] K. Kato, Lectures on the approach to Iwasawa theory of Hasse-Weil L-functions via $B_{\mathrm{dR}}$, Part I. In Arithmetical Algebraic Geometry (ed. E. Ballico), Lecture Notes in Math. 1553, Springer-Verlag, Berlin 1993, 50-163. Zbl 0815.11051 MR 1338860

[19] F. Knudsen and D. Mumford, The projectivity of the moduli space of stable curves I: Preliminaries on 'det' and 'Div'. Math. Scand. 39 (1976), 19-55. Zbl 0343.14008 MR 0437541

[20] S. Lichtenbaum, The Weil-étale topology on schemes over finite fields. Compositio Math. 141 (2005), 689-702. Zbl 1073.14024 MR 2135283

[21] S. Lang, Algebra. Revised third edition, Grad. Texts in Math. 211, Springer-Verlag, New York 2002. Zbl 0984.00001 MR 1878556

[22] B. Mazur and A. Wiles, Class fields of abelian extensions of $\mathbb{Q}$. Invent. Math. 76 (1984), 179-330. Zbl 0545.12005 MR 0742853

[23] K. Rubin, Global units and ideal class groups. Invent. Math. 89 (1987), 511-526. Zbl 0628.12007 MR 0903382

[24] K. Rubin, A Stark conjecture 'over $\mathbb{Z}$ ' for abelian $L$-functions with multiple zeros. Ann. Inst. Fourier 46 (1996), 33-62. Zbl 0834.11044 MR 1385509

[25] J. Tate, Les Conjectures de Stark sur les Fonctions L d'Artin en s=0. Progr. Math. 47, Birkhäuser, Boston, MA, 1984. Zbl 0545.12009 MR 0782485

[26] F. Thaine, On the ideal class groups of real abelian number fields, Ann. of Math. 128 (1988), 1-18. Zbl 0665.12003 MR 0951505

Received May 28, 2004

David Burns, King's College London, Department of Mathematics, Strand, London WC2R

2LS, England

E-mail: david.burns@kcl.ac.uk

Anthony Hayward, King's College London, Department of Mathematics, Strand, London

WC2R 2LS, England 